\title{
Bioinformatics clouds for big data manipulation
}

\author{
Lin Dai ${ }^{1,2}$, Xin Gao ${ }^{3}$, Yan Guo ${ }^{4}$, Jingfa Xiao ${ }^{1}$ and Zhang Zhang ${ }^{1 *}$
}

\begin{abstract}
As advances in life sciences and information technology bring profound influences on bioinformatics due to its interdisciplinary nature, bioinformatics is experiencing a new leap-forward from in-house computing infrastructure into utility-supplied cloud computing delivered over the Internet, in order to handle the vast quantities of biological data generated by high-throughput experimental technologies. Albeit relatively new, cloud computing promises to address big data storage and analysis issues in the bioinformatics field. Here we review extant cloud-based services in bioinformatics, classify them into Data as a Service (DaaS), Software as a Service (SaaS), Platform as a Service (PaaS), and Infrastructure as a Service (laaS), and present our perspectives on the adoption of cloud computing in bioinformatics.
\end{abstract}

Reviewers: This article was reviewed by Frank Eisenhaber, Igor Zhulin, and Sandor Pongor.

Keywords: Cloud computing, Bioinformatics, Big data, Data storage, Data analysis

\section{Background}

With significant advances in high-throughput sequencing technologies and consequently the exponential expansion of biological data, bioinformatics encounters difficulties in storage and analysis of vast amounts of biological data. The gap between sequencing throughput and computer capabilities in dealing with such big data is growing [1]. As reported on February 2012, two nanopore sequencing platforms (GridION and MinION) are capable of delivering ultra-long sequencing reads $(\sim 100 \mathrm{~kb})$ with additionally higher throughput and much lower cost [2]. This means that biological data will accumulate at an ever-faster pace. Digging out the "treasure" from massive biological data represents the primary challenge in bioinformatics, consequently placing unprecedented demands on big data storage and analysis. With the amount of data growing continuously, it is becoming increasingly daunting for small laboratories or even large institutions to establish and maintain computational infrastructures for data processing. At present, a promising solution to address this challenge is cloud computing [3-5], which exploits the full potential of multiple computers and delivers computation and storage as dynamically allocated virtual resources via the Internet [6].

\footnotetext{
* Correspondence: zhangzhang@big.ac.cn

${ }^{1}$ CAS Key Laboratory of Genome Sciences and Information, Beijing Institute of Genomics, Chinese Academy of Sciences, No.7 Beitucheng West Road, Building G, Chaoyang District, Beijing 100029, China

Full list of author information is available at the end of the article
}

\section{Review}

\section{Cloud computing as a public utility}

The term of "cloud computing" was inspired by the cloud symbol that is often employed to depict the Internet in flowcharts. As a matter of fact, cloud computing is not a new concept; it can date back to 1961 at the MIT Centennial when John McCarthy opined that "computation may someday be organized as a public utility" [7]. Cloud computing makes the best use of multiple computers to provide convenient and on-demand access to hosted resources (e.g., computation, storage, applications, servers, network) via Web Application Programming Interfaces (API). Due to its efficient and economical features, it is believed that cloud computing gains promise in transforming computing into a public utility [8]. Similar to extant public utilities (viz., water, electricity, gas, and telephone), computing utility packages a variety of computer resources as metered services ("pay-as-you-go"), which can be accessed by any person without the necessity to know where the services are hosted or how they are delivered. Whether a public utility or not, cloud computing has already become a significant technology in big data storage and analysis, exerting revolutionary influences on both academia and industry.

\section{Cloud-based resources in bioinformatics}

The popularization of cloud computing is to some extent attributable to open source software in aid of its implementation, such as Hadoop and associated software. Hadoop (http://hadoop.apache.org) features two key 
modules-MapReduce and Hadoop Distributed File System (HDFS). MapReduce divides a computational program into many small sub-problems and distributes them on multiple computer nodes, and HDFS provides a distributed file system that stores data on these nodes. Hadoop and its associated software are designed to handle load balancing among multiple nodes and to detect node failures that can be automatically re-executed on any node. In brief, Hadoop allows for the distributed processing of large datasets across multiple computer nodes, supports big data scaling (HDFS, HBase), and enables fault-tolerant parallelized analysis (MapReduce). Thus, Hadoop meets the needs of bioinformatics and several studies have successfully used Hadoop in bioinformatics [9-11], accordingly leading to cloud-based bioinformatics resources. As mentioned, cloud computing delivers hosted services over the Internet. Thus, bioinformatics clouds involve a large variety of services from data storage, data acquisition, to data analysis, which in general fall into four categories (Figure 1), viz., Data as a Service, Software as a Service, Platform as a Service, and Infrastructure as a Service [12]. In what follows, we summarize existing cloudbased resources in bioinformatics and classify them into these four categories (Table 1).

\section{Data as a service}

Bioinformatics clouds are heavily dependent on data, as data are fundamentally crucial for downstream analyses and knowledge discovery. It is reported that annual worldwide sequencing capacity is currently beyond $13 \mathrm{Pbp}$ and still on the increase annually by a factor of five (http://sourceforge.net/apps/mediawiki/jnomics). Due to such unprecedented growth in biological data, delivering Data as a

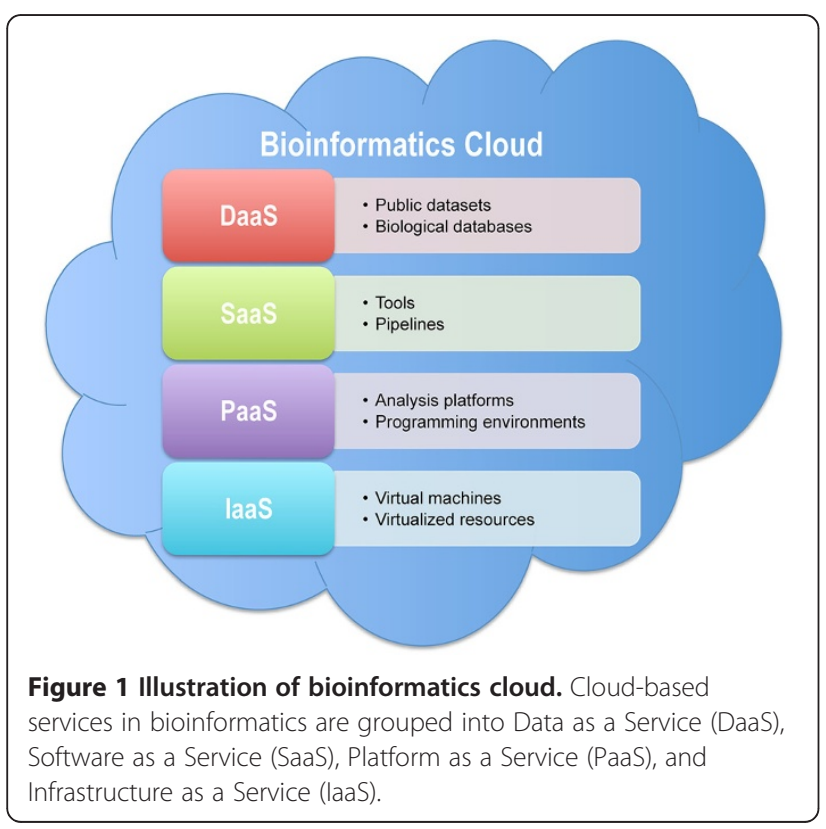

Service (DaaS) via the Internet is of utmost importance $[13,14]$. DaaS enables dynamic data access on demand and provides up-to-date data that are accessible by a wide range of devices that are connected over the Web. A case in point is Amazon Web Services (AWS) which provides a centralized repository of public data sets, including archives of GenBank, Ensembl, 1000 Genomes, Model Organism Encyclopedia of DNA Elements, Unigene, Influenza Virus, etc. As a matter of fact, AWS contains multiple public datasets for a variety of scientific fields, such as biology, astronomy, chemistry, climate, economics, etc. (http://aws. amazon.com/publicdatasets). All public datasets in AWS are delivered as services and thus can be seamlessly integrated into cloud-based applications [15].

\section{Software as a service}

Bioinformatics requires a large variety of software tools for different types of data analyses. Software as a Service (SaaS) delivers software services online and facilitates remote access to available bioinformatics software tools through the Internet. As a consequence, SaaS eliminates the need for local installation and eases software maintenances and updates, providing up-to-date cloud-based services for bioinformatic data analysis over the Web. In the past several years, efforts have been made to develop cloud-scale tools, including sequence mapping [16-18], alignment [19], assembly (Contrail, Gaea and Hecate; unpublished), expression analysis [20-22], sequence analysis (Jnomics; unpublished), orthology detection [23], peak caller for ChIP-seq data [24], functional annotation of variants from multiple personal genomes [25], identification of epistatic interactions of single nucleotide polymorphisms (SNPs) [26], and various cloud-based applications for NGS (Next-Generation Sequencing) data analysis (BGI Cloud and EasyGenomics; unpublished).

\section{Platform as a service}

To make the cloud programmable, Platform as a Service (PaaS) offers an environment for users to develop, test and deploy cloud applications where computer resources scale automatically and dynamically to match application demand, so that users do not need to know how many resources are required or to assign resources manually in advance. PaaS features rapid application development and good scalability, presenting usefulness in developing specific applications for big biological data analysis. Typically, the environment delivered by PaaS includes programming language execution environments, web servers, and databases. From this point, by delivering data as a service and functioning as a database, DaaS can be regarded as an enhancement of PaaS. Currently, there are only two PaaS platforms in bioinformatics belonging to web servers, namely, Eoulsan [27], which is a cloud-based platform for high-throughput sequencing analyses, and 
Table 1 Cloud resources in bioinformatics

\begin{tabular}{|c|c|}
\hline Resource & Description \& availability \\
\hline \multicolumn{2}{|c|}{ Data as a Service (DaaS): } \\
\hline AWS Public Datasets & $\begin{array}{l}\text { Cloud-based archives of GenBank, Ensembl, } 1000 \text { Genomes, Model Organism Encyclopedia of DNA Elements, Unigene, } \\
\text { Influenza Virus, etc.; http://aws.amazon.com/publicdatasets }\end{array}$ \\
\hline \multicolumn{2}{|c|}{ Software as a Service (SaaS): } \\
\hline $\begin{array}{l}\text { BGI Cloud } \\
\text { (unpublished) }\end{array}$ & Cloud-based implementations of various genomic analysis applications; http://cloud.genomics.cn \\
\hline CloudAligner [16] & Fast and full-featured MapReduce-based tool for sequence mapping; http://cloudaligner.sourceforge.net \\
\hline CloudBLAST [19] & A cloud-based implementation of NCBI BLAST; http://ammatsun.acis.ufl.edu/amwiki/index.php/CloudBLAST_Project \\
\hline CloudBurst [17] & Highly sensitive short read mapping with MapReduce; http://cloudburst-bio.sourceforge.net \\
\hline Contrail (unpublished) & Cloud-based de novo assembly of large genomes; http://contrail-bio.sourceforge.net \\
\hline Crossbow [18] & Read Mapping and SNP calling using cloud computing; http://bowtie-bio.sf.net/crossbow \\
\hline $\begin{array}{l}\text { EasyGenomics } \\
\text { (unpublished) }\end{array}$ & $\begin{array}{l}\text { Cloud-based NGS pipelines for whole genome resequencing, exome resequencing, RNA-Seq, small RNA and de novo } \\
\text { assembly; http://www.easygenomics.org }\end{array}$ \\
\hline eCEO [26] & $\begin{array}{l}\text { Cloud-based identification of large-scale epistatic interactions in genome-wide association study (GWAS); http://www.comp. } \\
\text { nus.edu.sg/ wangzk/eCEO.html }\end{array}$ \\
\hline FX [20] & RNA-Seq analysis tool; http://fx.gmi.ac.kr \\
\hline Gaea (unpublished) & Cloud-based genome re-sequencing assembly; http://bgiamericas.com/data-analysis/cloud-computing \\
\hline Hecate (unpublished) & Cloud-based de novo assembly; http://bgiamericas.com/data-analysis/cloud-computing \\
\hline Jnomics (unpublished) & Cloud-scale sequence analysis suite based on Apache Hadoop; http://sourceforge.net/apps/mediawiki/jnomics \\
\hline Myrna [21] & Differential gene expression tool for RNA-Seq; http://bowtie-bio.sourceforge.net/myrna \\
\hline PeakRanger [24] & Cloud-enabled peak caller for ChIP-seq data; http://www.modencode.org/software/ranger \\
\hline RSD [23] & $\begin{array}{l}\text { Reciprocal smallest distance algorithm for ortholog detection using Amazon's Elastic Computing Cloud; http://roundup.hms } \\
\text { harvard.edu }\end{array}$ \\
\hline VAT [25] & $\begin{array}{l}\text { Variant annotation tool to functionally annotate variants from multiple personal genomes at the transcript level; http://vat. } \\
\text { gersteinlab.org }\end{array}$ \\
\hline YunBe [22] & Pathway-based or gene set analysis of expression data; http://tinyurl.com/yunbedownload \\
\hline \multicolumn{2}{|c|}{ Platform as a Service (PaaS): } \\
\hline Eoulsan [27] & Cloud-based platform for high throughput sequencing analyses; http://transcriptome.ens.fr/eoulsan \\
\hline Galaxy Cloud [28,29] & Cloud-scale Galaxy for large-scale data analysis; http://galaxy.psu.edu \\
\hline \multicolumn{2}{|c|}{ Infrastructure as a Service (laaS): } \\
\hline Cloud BioLinux [30] & $\begin{array}{l}\text { A publicly accessible virtual machine for high performance bioinformatics computing using cloud platforms; http:// } \\
\text { cloudbiolinux.org }\end{array}$ \\
\hline ClovR [31] & A portable virtual machine for automated sequence analysis using cloud computing; http://clovr.org \\
\hline
\end{tabular}

Galaxy Cloud [28,29], which is a cloud-scale Galaxy for large-scale data analyses.

\section{Infrastructure as a service}

To reach the full potential of computer resources, Infrastructure as a service (IaaS) offers a full computer infrastructure by delivering all kinds of virtualized resources via the Internet, including hardware (e.g., CPUs) and software (e.g., operating systems). Users can access virtualized resources as a public utility and pay for the cloud resources that they utilize. Since different users often need different cloud resources, flexibility and customization are essential to IaaS. With the ongoing and rapid advancement of IT, it is increasingly efficient to run applications within Virtual Machines (VMs). VM isolates users from the underlying infrastructure and provides flexibility to meet the customized needs of different users. To date, there are two examples of IaaS in bioinformatics, viz., Cloud BioLinux [30], which is a publicly accessible VM for high-performance bioinformatics computing, and CloVR [31], which is a portable VM that incorporates several pipelines for automated sequence analysis.

\section{Toward bioinformatics clouds}

Albeit relatively new, cloud computing holds great promise in effectively addressing big data storage and analysis problems in bioinformatics. Below, we present our perspectives on the adoption of cloud computing in bioinformatics research.

\section{Placing data and software into the cloud}

The traditional way for bioinformatics analysis often involves downloading data from public sites (e.g., NCBI, Ensembl), installing software tools locally, and running 
analyses on in-house computer resources. By placing data and software into the cloud and delivering them as services (namely, DaaS and SaaS), data and software can be seamlessly and easily integrated into the cloud so as to achieve big data storage and analysis. Thus, it would be desirable to store and analyze big biological data within the cloud.

In the era of big data, however, only a tiny amount of biological data is accessible in the cloud at present (only AWS, including GenBank, Ensembl, 1000 Genomes, etc.) and the vast majority of data are still deposited in conventional biological databases. In the long run, more and more sequencing projects, such as the Genome 10K Project (a collection of DNA sequences representing the genomes of 10,000 vertebrate species; http://www.genome10k.org), the 1001 Genomes Project (a catalog of genetic variation in 1001 strains of Arabidopsis thaliana; http://www.1001genomes.org), the 1KITE Project (1K Insect Transcriptome Evolution; http://www.1kite.org), TCGA (the Cancer Genome Atlas; http://cancergenome.nih.gov), etc., would generate ultra-large volumes of biological data and thus require bioinformatics clouds for big data storage, sharing and analysis [32]. In addition, to decipher the most important and complex biological questions often involves the utilization of multiple tools [33]. However, extant efforts have only touched a small fraction of cloud-based tools. Most software tools are written for desktop (rather than cloud) [34] and therefore are not provided as cloud-based web services accessible via the Web, making it infeasible to perform complex bioinformatics tasks. To fulfill big data storage, sharing and analysis with lower cost and higher efficiency, it is essentially required that a large number of biological data as well as a wide variety of bioinformatics tools should be publicly accessible in the cloud and delivered as services through the Internet. However, it should be also noted that biology is in its infancy (compared with other disciplines, e.g., physics) and many theoretical problems in biology are under the surface, albeit a huge volume of biological data are available now. To address theoretical problems and uncover fundamental theories in biology, it often involves hypothesis formulation, experiment design, data generation/collection, tool development (for data analysis), and knowledge formalization, which can be helpful to recognize what data and tools should be placed into the cloud.

\section{Big data transfer}

Transferring vast amounts of biological data to the cloud is a significant bottleneck in cloud computing [6]; it is not unusual at present to physically ship hard drives to the cloud center (http://aws.amazon.com/importexport). Currently, a promising solution is to integrate innovative transferring technologies into cloud computing. One example is the cloud-based EasyGenomics (http://www. genomics.cn/en/news/show_news?nid=99014), released by BGI (Beijing Genomics Institute), that achieves highspeed genomic data delivery by Aspera's fasp ${ }^{\mathrm{TM}}$ highspeed file transfer technology (which dramatically speeds file transfers over the Web and outperforms conventional technologies such as FTP and HTTP; http://www. asperasoft.com/en/technology/fasp_overview_1/fasp_ technology_overview_1). In June 2012, BGI succeeded in transferring genomic data across the Pacific Ocean at a sustained rate of almost 10 Gigabits per second (http:// www.bio-itworld.com/news/06/29/12/Data-tsunami-BGItransfers-genomic-data-Pacific-10-gigabits-second.html), demonstrating that high-speed transfer technologies (such as Aspera's fasp) are capable of dealing with big data transfers over the Web. Aside from high-speed transfer technologies, there are other technologies that can also aid big data transfer, such as data compression $[35,36]$ and Peer-to-Peer (P2P) data distribution [37,38].

\section{A cloud-based lightweight programming environment}

To automate data analysis, bioinformatic tasks are often implemented as pipelines by linking the output of one tool with the input of another. To perform large-scale data analysis and aid in the development of corresponding bioinformatic pipelines, a cloud-based lightweight programming environment is needed, which allows the swift development of customized pipelines from a large pool of tools and enables automated and configurable analysis on the cloud. Currently, the cloud-based programming paradigm adopted in the bioinformatics community is Hadoop [11,16,17,20,27], in which computation-consuming and data-intensive analyses are primarily solved by distributing tasks over multiple nodes (see tutorials at http://wiki.apache.org/hadoop). However, substantial computational skills are still required for developing cloud-based pipelines in Hadoop and its programming environment is not lightweight for most biologists or people with no or limited programming experience. Ideally, this would be a lightweight programming environment that does not require extensive coding by keyboard. Rather, it would be done easily by mouse, viz., "drag+drop". Such an environment also features remote access to diverse types of resources based on utility-supplied cloud computing, consisting well with the potential trend of e-Science [39] (that is, scientific research in many disciplines is carried out via the internet). Furthermore, when building such an environment, attention should be paid to setting up a system of standards for data exchanges among different software tools [40], which can in return pave the way for realizing the full potential of lightweight programming environment.

\section{Open bioinformatics clouds}

Incentivized by the potential big profits to be made on a pay-as-you-go basis, there are multiple cloud providers 
and it is predicted that in the following years there will be more providers, building industrial or academic, private or public clouds. Currently, the largest provider is Amazon, which provides commercial clouds for processing big data. Additionally, Google also provides a cloud platform (https://cloud.google.com) that allows users to develop and host web applications, and store and analyze data. However, commercial clouds are currently not yet able to provide ample data and software for bioinformatics analysis. Moreover, it is very difficult for commercial clouds to keep pace with the emerging needs from academic research, consequently requiring specific clouds for bioinformatics studies. It goes without saying that open access and public availability of data and software are of great significance to science [41]. When data and software are all in the cloud, keeping the cloud open and publicly available to the scientific community is essential for bioinformatics research [42]. Therefore, it is most likely that future efforts should be devoted to building open bioinformatics clouds and providing public access to the scientific community. The potential resulting benefits of such bioinformatics clouds include easing large-scale data integration, enabling repeatable and reproducible analyses, maximizing the scope for sharing, and harnessing collective intelligence for knowledge discovery. With the presence of numerous bioinformatics clouds, interoperability and standardization between clouds will become important issues $[43,44]$.

\section{Conclusions}

Here we reviewed extant cloud-based resources in bioinformatics and classified them into DaaS, SaaS, PaaS, and IaaS. Since cloud computing bears great promise in effectively addressing big data storage and analysis, future efforts in building bioinformatics clouds involve developing a large variety of services from data storage, data acquisition, to data analysis, accordingly making utility-supplied cloud computing delivered over the Internet. In the era of big data, bioinformatics clouds should integrate both data and software tools, equip with high-speed transfer technologies and other related technologies in aid of big data transfer, provide a lightweight programming environment to help people develop customized pipelines for data analysis, and most important, be open and publicly accessible to the whole scientific community.

\footnotetext{
Abbreviations

DaaS: Data as a service; SaaS: Software as a service; PaaS: Platform as a service; laaS: Infrastructure as a service; API: Application programming interface; AWS: Amazon web service; VM: Virtual machine.
}

\section{Competing interests}

The authors declare that they have no competing interests.

\section{Authors' contributions}

LD drafted the manuscript. XG, JX, and YG revised the manuscript critically for important intellectual content. ZZ conceived of the study, supervised the project, and revised the manuscript. All authors read and approved the final manuscript.

\section{Reviewers' comments}

Reviewer 1: Dr. Frank Eisenhaber (Bioinformatic Institute, Singapore) The report by Dai et al. is a timely, well structured review of the opportunities associated with cloud computing in the bioinformatics domain and provides insight both into the existing state of the art and near-future possibilities. As such, it appears useful for the community.

At the same time, nothing is said to which extent these development have already contributed to new biological insight. Do the authors have examples for this?

Authors' response: Yes, Galaxy is a case in point; it is widely used for a wide range of bioinformatics analyses and cited by more than 300 times.

There is the danger that the excitement with large data and with making it available shadows the actual reason why this data is recorded. Many of these so-called high impact, recently published OMICS papers are actually a boring reading with no new idea (even at the methodical level) and no new biology discovered. At the end, it is about biomolecular mechanisms that need to be known.

Authors' response: We fully agree. To reflect this point more clearly, we expanded our description accordingly. Albeit currently we have high-throughput sequencers and large-scale data, biology is likened to the $16^{\text {th }}$ century physics in its state of development, lacking fundamental rules and theories, and many theoretical problems in biology are still under the surface. To address theoretical problems and uncover fundamental theories in biology, it often involves hypotheses formulation, experiment design, data generation/collection, tool development (for data analysis), and knowledge formalization, which can be helpful to recognize what data and tools should be placed into the cloud. The large data stream today is, to a great extent, due to the infantile methodology of measuring sequences and expression profiles. The TBs and PBs of sequencing data can be condensed to GBs with post-experimental processing and it are these GBs that are the actual object of scientific analysis. The human genome sequence per individual is just a few GB and, most likely and hopefully:-), it will not grow much in the future. Similarly, thousands of individual genomes might be stored as variations of a reference genome and this will, most likely, cost less than a GB per genome. Expression profiles are actually arrays of genomic locations and occurrence numbers and thus, are also in the GB range. Thus, the shift to big data science and the emphasis on new IT developments instead of doing the actual life science research might drift a considerable part of the community away into non-relevant efforts. To remember a historical example: There was a great public, large data effort with creating astronomic maps for solving the longitudinal problem in the 17th century Britain; yet, John Harrison solved it by constructing a clock.

Authors' response: Thank you for your excellent points, which we fully agree. As an interdisciplinary field, bioinformatics is influenced by any advance in IT or biology. Take Web technology as an example, in retrospect, Web 1.0 was "readonly" (50K average band width, ABW), Web 2.0 is "read-write" (1M ABW), and Web 3.0 promises to have the attributes of "read-write-execute" with greater $A B W[40]$, as cloud computing delivers all kinds of computer resources via the internet which to some extent aids the furtherance of Web 3.0. The rapid advancement of information technology (e.g., Web technology) brings a lot to bioinformatics, including data analysis, data storage, and data sharing. Thus, it is likely inevitable that there will be a shift to "big" data science based on utilitysupplied cloud computing, which is consistent with the potential trend of eScience [39] (that is, scientific research in many disciplines is carried out via the internet). At the same time, we completely agree that "big" data to some degree result from lacks of fundamental theories; these theories can guide us in developing methods and formulating hypotheses and then know what data should be stored. Our perspectives are that in the future more and more computer scientists will help biologists build a light-weighted programming environment for biological data analysis and collaborate with biologists to conduct actual life science research. We clarified the corresponding description and added a related citation (Ref [39]) in the revised version. Reviewer 2: Dr. Igor Zhulin (University of Tennessee, United States of America) The review summarizes advantages of using cloud computing for "big data" storage and analysis issues in bioinformatics. In general, it does a fair job on 
this front. However, disadvantages of clouds are not discussed in this review at all. For example, time-critical calculations, complex tasks that require data management (load balancing, fault tolerance issues, etc.) will not do well on clouds that lack the edge of advanced HPC architectures.

Authors' response: Thanks for your valuable comments. We accepted your comments and added some description in the main text. Hadoop (http:// hadoop.apache.org) features two key modules-MapReduce and Hadoop Distributed File System (HDFS). MapReduce divides a computational program into many small sub-problems and distributes them on multiple computer nodes, and HDFS provides a distributed file system that stores data on these nodes. Hadoop and its associated software are designed to handle load balancing among multiple nodes and to detect node failures that can be automatically re-executed on any node. Therefore, Hadoop is capable of performing time-critical calculations by distributing tasks and large datasets over multiple computer nodes, supporting big data scaling, and enabling faulttolerant parallelized analysis.

Reviewer 3: Prof. Sandor Pongor (International Centre for Genetic Engineering and Biotechnology, Italy)

I am not an expert of cloud computing but I am am very curious to see the potentials of this technology for bioinformatics. I found the paper clearly written, concise and well structured - it gives good introduction to this field, that readers of this journal will appreciate in my opinion. What I kind of miss is the user's perspective. When shall a student or a user of bioinformatics think about a cloud solution? Where to get introductory texts? On a more practical side: Teaching the bioinformatics of big data (high throughput) seems to be easy by putting a virtual server on the cloud ... Where are the could based courses? These are simple questions and the authors may want to dedicate a little space to dealing with them.

Authors' response: Thank you for your thoughtful and excellent comments. "When shall a student or a user of bioinformatics think about a cloud solution", is highly dependent on the user's need and the computer resources the user has. Taking Saas as an example, if a computational task is very time-consuming and also this task can be divided into many small sub-tasks, it might be better to put this task into the cloud and to run it as Saas. The cloud just mentioned can be offered by a cloud provider (e.g., Amazon), or be in-house, self-made which makes full use of multiple computers available in one lab/institution. The relevant introductory texts are available at http://wiki.apache.org/hadoop, which provides general information and tutorials on how to build a Hadoop-based cloud and includes several practical examples. There is an online course- "Cloud Application Development" at http://my.ss.sysu.edu.cn/cloud/, which also contains other related resources and references. In addition, Galaxy (https:// main.g2.bx.psu.edu) is capable of performing interactive data analyses, which can serve as an E-learning platform for students.

\section{Acknowledgements}

We thank Dr. Jun Yu and Ang Li for their valuable comments on this work. This work was supported by grants from King Abdullah University of Science and Technology (a start-up grant to XG), the National Natural Science Foundation of China (60803050 and 61132009 to LD), the "100-Talent Program" of Chinese Academy of Sciences (Y1SLXb1365 to ZZ), the National Basic Research Program (973 Program; 2010CB126604 to JX) and the National Programs for High Technology Research and Development (863 Program; 2012AA020409 to JX and ZZ), the Ministry of Science and Technology of the People's Republic of China.

\section{Author details}

${ }^{1}$ CAS Key Laboratory of Genome Sciences and Information, Beijing Institute of Genomics, Chinese Academy of Sciences, No.7 Beitucheng West Road, Building G, Chaoyang District, Beijing 100029, China. ${ }^{2}$ School of Computer Science and Technology, Beijing Institute of Technology, Beijing 100081, China. ${ }^{3}$ Computer, Electrical and Mathematical Sciences and Engineering Division, King Abdullah University of Science and Technology (KAUST), Thuwal 23955-6900, Saudi Arabia. ${ }^{4}$ Cloud Development and Cloud Solution Integration, IBM China Systems \& Technology Lab, IBM Co. Ltd, Beijing 100193, China.

Received: 19 September 2012 Accepted: 26 November 2012 Published: 28 November 2012

\section{References}

1. Schatz MC, Langmead B, Salzberg SL: Cloud computing and the DNA data race. Nat Biotechnol 2010, 28(7):691-693.

2. Eisenstein $\mathrm{M}$ : Oxford Nanopore announcement sets sequencing sector abuzz. Nat Biotechnol 2012, 30(4):295-296.

3. Schadt EE, Linderman MD, Sorenson J, Lee L, Nolan GP: Cloud and heterogeneous computing solutions exist today for the emerging big data problems in biology. Nat Rev Genet 2011, 12(3):224.

4. Schadt EE, Linderman MD, Sorenson J, Lee L, Nolan GP: Computational solutions to large-scale data management and analysis. Nat Rev Genet 2010, 11(9):647-657.

5. Grossman RL, White KP: A vision for a biomedical cloud. J Intern Med 2012, 271(2):122-130.

6. Armbrust M, Fox A, Griffith R, Joseph AD, Katz RH, Konwinski A, Lee G, Patterson DA, Rabkin A, Stoica I, et al: Above the Clouds: A Berkeley View of Cloud Computing. Berkeley: EECS Department, University of California; 2009.

7. Garfinkel SL: Architects of the Information Society: Thirty-Five Years of the Laboratory for Computer Science at MIT. Cambridge, MA: The MIT Press; 1999.

8. Buyya R, Yeo CS, Venugopal S, Broberg J, Brandic I: Cloud computing and emerging IT platforms: vision, hype, and reality for delivering computing as the 5th utility. Future Gener Comp Sy 2009, 25(6):599-616.

9. Dudley JT, Butte AJ: In silico research in the era of cloud computing. Nat Biotechnol 2010, 28(11):1181-1185.

10. Stein LD: The case for cloud computing in genome informatics. Genome Biol 2010, 11(5):207.

11. Taylor RC: An overview of the Hadoop/MapReduce/HBase framework and its current applications in bioinformatics. BMC Bioinformatics 2010, 11(Suppl 12):S1.

12. Stanoevska-Slabeva K, Wozniak T: Cloud Basics - An Introduction to Cloud Computing. In Grid and Cloud Computing: Business Perspective on Technology and Applications. Edited by Stanoevska K, Wozniak T, Ristol S. Berlin: Springer; 2010:47-61.

13. Truong HL, Dustdar S: On Analyzing and Specifying Concerns for Data as a Service. In 2009 leee Asia-Pacific Services Computing Conference (Apscc 2009). 2009:83-90.

14. DaaS: The New Information Goldmine. http://online.wsj.com/article/ SB125071202052143965.html

15. Fusaro VA, Patil P, Gafni E, Wall DP, Tonellato PJ: Biomedical cloud computing with Amazon Web Services. PLoS Comput Biol 2011, 7(8):e1002147.

16. Nguyen T, Shi W, Ruden D: CloudAligner: a fast and full-featured MapReduce based tool for sequence mapping. BMC Res Notes 2011, 4:171.

17. Schatz MC: CloudBurst: highly sensitive read mapping with MapReduce. Bioinformatics 2009, 25(11):1363-1369.

18. Langmead B, Schatz MC, Lin J, Pop M, Salzberg SL: Searching for SNPs with cloud computing. Genome Biol 2009, 10(11):R134.

19. Matsunaga A, Tsugawa M, Fortes J: Combining MapReduce and Virtualization on Distributed Resources for Bioinformatics Applications. In Fourth IEEE International Conference on eScience; 2008:222-229.

20. Hong D, Rhie A, Park SS, Lee J, Ju YS, Kim S, Yu SB, Bleazard T, Park HS, Rhee $H$, et al: FX: an RNA-Seq analysis tool on the cloud. Bioinformatics 2012, 28(5):721-723.

21. Langmead $B$, Hansen KD, Leek JT: Cloud-scale RNA-sequencing differential expression analysis with Myrna. Genome Biol 2010, 11(8):R83.

22. Zhang L, Gu S, Liu Y, Wang B, Azuaje F: Gene set analysis in the cloud. Bioinformatics 2012, 28(2):294-295.

23. Wall DP, Kudtarkar P, Fusaro VA, Pivovarov R, Patil P, Tonellato PJ: Cloud computing for comparative genomics. BMC Bioinformatics 2010, 11:259.

24. Feng X, Grossman R, Stein L: PeakRanger: a cloud-enabled peak caller for ChIP-seq data. BMC Bioinformatics 2011, 12:139.

25. Habegger L, Balasubramanian S, Chen DZ, Khurana E, Sboner A, Harmanci A, Rozowsky J, Clarke D, Snyder M, Gerstein M: VAT: a computational framework to functionally annotate variants in personal genomes within a cloud-computing environment. Bioinformatics 2012. Epub ahead of print.

26. Wang Z, Wang Y, Tan KL, Wong L, Agrawal D: eCEO: an efficient Cloud Epistasis cOmputing model in genome-wide association study. Bioinformatics 2011, 27(8):1045-1051.

27. Jourdren L, Bernard M, Dillies M-A, Le Crom S: Eoulsan: a cloud computing-based framework facilitating high throughput sequencing analyses. Bioinformatics 2012. doi:2010.1093/bioinformatics/bts2165. published online April 5, 2012. 
28. Afgan E, Baker D, Coraor N, Goto H, Paul IM, Makova KD, Nekrutenko A, Taylor J: Harnessing cloud computing with Galaxy Cloud. Nat Biotechnol 2011, 29(11):972-974.

29. Afgan E, Baker D, Coraor N, Chapman B, Nekrutenko A, Taylor J: Galaxy CloudMan: delivering cloud compute clusters. BMC Bioinformatics 2010, 11(Suppl 12):S4

30. Krampis K, Booth T, Chapman B, Tiwari B, Bicak M, Field D, Nelson K: Cloud BioLinux: pre-configured and on-demand bioinformatics computing for the genomics community. BMC Bioinformatics 2012, 13(1):42.

31. Angiuoli SV, Matalka M, Gussman A, Galens K, Vangala M, Riley DR, Arze C, White JR, White O, Fricke WF: CloVR: a virtual machine for automated and portable sequence analysis from the desktop using cloud computing. BMC Bioinformatics 2011, 12:356.

32. Dudley JT, Pouliot Y, Chen R, Morgan AA, Butte AJ: Translational bioinformatics in the cloud: an affordable alternative. Genome Med 2010, 2(8):51.

33. Zhang Z, Bajic VB, Yu J, Cheung K-H, Townsend JP: Data Integration in Bioinformatics: Current Efforts and Challenges. In Bioinformatics - Trends and Methodologies. Edited by Mahdavi MA. Rijeka, Croatia: InTech - Open Access Publisher; 2011.

34. Fox A: Cloud computing-what's in it for me as a scientist? Science 2011, 331(6016):406-407.

35. Deorowicz S, Grabowski S: Compression of DNA sequence reads in FASTQ format. Bioinformatics 2011, 27(6):860-862.

36. Cox AJ, Bauer MJ, Jakobi T, Rosone G: Large-scale compression of genomic sequence databases with the Burrows-Wheeler transform. Bioinformatics 2012, 28(11):1415-1419.

37. Langille MGl, Eisen JA: BioTorrents: a file sharing service for scientific data. PLoS One 2010, 5(4):e10071.

38. Sangket U, Phongdara A, Chotigeat W, Nathan D, Kim WY, Bhak J, Ngamphiw C, Tongsima S, Khan AM, Lin H, et al: Automatic synchronization and distribution of biological databases and software over low-bandwidth networks among developing countries. Bioinformatics 2008, 24(2):299-301.

39. Bishop M: e-Science. Brief Bioinform 2003, 4(3):208-209.

40. Zhang Z, Cheung KH, Townsend JP: Bringing Web 2.0 to bioinformatics. Brief Bioinform 2009, 10(1):1-10.

41. Marx V: My data are your data. Nat Biotechnol 2012, 30(6):509-511.

42. Rosenthal A, Mork P, Li MH, Stanford J, Koester D, Reynolds P: Cloud computing: a new business paradigm for biomedical information sharing. J Biomed Inform 2010, 43(2):342-353.

43. Dillon T, Wu C, Chang E: Cloud Computing: Issues and Challenges. In Int Con Adv Info Net; 2011:27-33.

44. Parameswaran AV, Chaddha A: Cloud interoperability and standardization. SETLabs Briefings 2009, 7(7):19-26.

doi:10.1186/1745-6150-7-43

Cite this article as: Dai et al:: Bioinformatics clouds for big data manipulation. Biology Direct 2012 7:43.

\section{Submit your next manuscript to BioMed Central and take full advantage of:}

- Convenient online submission

- Thorough peer review

- No space constraints or color figure charges

- Immediate publication on acceptance

- Inclusion in PubMed, CAS, Scopus and Google Scholar

- Research which is freely available for redistribution

Submit your manuscript at www.biomedcentral.com/submit
() Biomed Central 